\title{
ADICIONES Y NOTAS A LA FLORA DEL PARQUE NACIONAL LLULLAILLACO, II REGION, CHILE
}

\section{ADDITIONS AND NOTES TO THE FLORA OF LLULLAILLACO NATIONAL PARK, REGION OF ANTOFAGASTA,NORTHERN CHILE}

\author{
Alicia Marticorena ${ }^{1}$, Verónica Pardo ${ }^{2}$, Alejandro Peñaloza ${ }^{2}$, María A. Negritto ${ }^{1}$, \\ Lohengrin A. Cavieres ${ }^{1} \&$ Mario Parada ${ }^{3}$ \\ ${ }^{1}$ Departamento de Botánica, Facultad de Ciencias Naturales y Oceanográficas, Universidad de Concepción, Casilla \\ 160-C, Concepción, Chile. \\ ${ }^{2}$ Centro de Ecología Aplicada Ltda. (CEA), Av. Suecia 3304, Ñuñoa, Santiago. \\ ${ }^{3}$ Minera Escondida Ltda., Av. de la Minería No501, Antofagasta.
}

\begin{abstract}
RESUMEN
Se presentan nuevos registros de 16 especies, 10 géneros y 1 familia para la flora del Parque Nacional Llullaillaco (Región de Antofagasta). Con esto la flora total del parque se eleva a 126 especies, contenidas en 74 géneros y 31 familias. El número de especies endémicas en el PN Llullaillaco se eleva a 21 (16,6\%). Este porcentaje de endemismo es uno de los más altos documentados para un parque nacional ubicado en los Andes de Chile. Además, se señalan algunos comentarios nomenclaturales para algunas especies previamente documentadas en otros estudios.
\end{abstract}

Palabras ClaVes: Endemismos, flora altoandina, nuevas citas.

\section{ABSTRACT}

New records for 16 species, 10 genera and one family are reported for the flora of the Llullaillaco National Park (Region of Antofagasta) in northern Chile. The total known flora of this National Park is thus increased to 126 species, comprising 74 genera and 31 families. The number of endemic species in Llullaillaco National Park is increased to 21, indicating that the incidence of endemics ( $16.6 \%$ of its flora) is one of the highest found for a national park in the Chilean Andes. We also include nomenclature comments on some species previously reported in other studies.

KEYwords: Andean flora, endemism, new records.

\section{INTRODUCCION}

El Parque Nacional Llullaillaco se encuentra en los Andes del norte de Chile (Región de Antofagasta), entre las latitudes $24^{\circ} 30^{\prime} \mathrm{S}-25^{\circ} 10^{\circ} \mathrm{S}$ y las longitudes $68^{\circ} 30^{\circ} \mathrm{W}-69^{\circ} 15^{\circ} \mathrm{W}$. La altitud del parque varía entre los $3500 \mathrm{~m}$ s.n.m.y la cumbre del volcán Llullaillaco a $6750 \mathrm{~m}$. La zona donde actualmente se ubica el parque ha sido explorada botánicamente desde 1860 con el viaje del naturalista alemán R. A. Philippi. Desde entonces, hasta la última revisión de la flora vascular del parque realizada por Luebert \& Gajardo (1999), se habían registrado 110 especies de plantas vasculares, pertenecientes a 63 géneros y 30 familias. No obstante, el parque se ubica en una zona de transición entre las lluvias de invierno y verano (Arroyo et al. 1998), con grandes fluctuaciones interanuales en las precipitaciones (Huston \& Hartley 2003), y es posible que algunas especies no emerjan en períodos climáticamente desfavorables. Por lo tanto, a pesar de las recolecciones anteriores, es factible que aún se encuentren especies no reportadas. 
Durante el período de enero de 2001 a abril de 2004, se realizaron campañas de recolección en el parque, las que han permitido ampliar el número de especies conocidas. En este trabajo damos a conocer dichas especies, incluyendo además comentarios sobre algunos problemas nomenclaturales detectados en trabajos anteriores sobre la flora del Parque Nacional Llullaillaco.

\section{ANGIOSPERMAE: MAGNOLIOPSIDA APIACEAE}

\section{Azorella trifoliolata Clos}

Planta en rosetas laxas, de hasta $15 \mathrm{~cm}$ de altura y diámetro. Hojas ternadas a ternado-pinnatisectas, segmentos linear-oblongos a linear-lanceolados, agudos, pecíolos delgados. Umbelas con 10-45 flores; flores blanco-amarillentas. Fruto anchamente ovoide, dorsalmente comprimido.

Escasa en las vegas. En Chile, crece en las III-RM y VIII-XII Región, también en Argentina. Exs.: Río Frío, 3830 m, enero 2004, V. Pardo 107 (CONC 158163).

\section{AsteraceAe}

\section{Baccharis santelicis Phil. subsp. santelicis}

Arbusto dioico, de hasta $100 \mathrm{~cm}$ de alto, resinoso, muy ramoso. Ramas cicatricosas, glutinosas. Hojas pequeñas, angostamente ovadas a espatuladas, enteras o a veces con un diente a cada lado, ápice redondeado. Capítulos solitarios en la axila de las hojas subterminales, con brácteas involucrales dispuestas en 3-5 series.

Abundancia media. Crece también en la I Región, y en Argentina, Perú y Bolivia. Exs.: Quebrada Zorras, $4000 \mathrm{~m}$, febrero 2001, V. Pardo 74 (CONC 158147).

\section{Polyachyrus carduoides Phil.}

Planta hirsuto-glandulosa, aromática. Hojas agrupadas en la parte inferior y media, linear-oblongas, irregularmente pinnatifidas, espinudo-dentadas, hirsuto-glandulosa, base con 2 orejuelas dentadas. Flores en capítulos globosos.

Escasa en borde de vega. Endémica de Chile, se la encuentra de la I-IV Región. Exs.: Quebrada Zorras, 3445 m, enero 2004, V. Pardo 80 (CONC 158160).

\section{Senecio haenkei DC.}

Sufrútice ramoso, albo-tomentoso, de $30-50 \mathrm{~cm}$ de altura. Hojas lanceoladas, alternas, de 2-8 cm, ápice agudo, margen entero, revoluto. Cimas corimbiformes con 3-15 capítulos discoideos. Involucro acampanado, con 10-14 brácteas. Flores numerosas, de color amarillo pálido.

Escasa. Crece entre la II-IV Región y en Argentina. Exs.: Quebrada Llullaillaco, $3230 \mathrm{~m}$, enero 2004, V. Pardo 114 (CONC 158165).

\section{BoraginaCEAE}

\section{Cryptantha diffusa (Phil.) I.M.Johnst.}

Hierba anual de hasta $20 \mathrm{~cm}$ de alto, hirsuta, con pelos blancos. Hojas lineares o estrechamente lanceoladas, híspidas. Flores dispuestas en cincinos. Cáliz con segmentos estrechamente ovados, hirsutos. Corola blanca, de 1,5-2,5 mm, con lóbulos redondeados. Clusas 4 ó 1-3 por aborto, blancogrisáceas o parduscas, ovoide-trígonas, granuladas a tuberculadas, con surcos transversales más o menos sinuosos que le dan un aspecto cerebroide.

Escasa. Habita entre la I-IV Región, y en Argentina y Bolivia. Exs.: Quebrada Zorras, $4050 \mathrm{~m}$, noviembre 2002, V. Pardo 6 (CONC 158148).

\section{Caryophyllaceae}

\section{Silene mandonii (Rohrb.) Bocquet}

Hierba perenne, erecta, hojas en roseta, pubescente. Hojas lanceoladas, sésiles. Flores solitarias, pedunculadas, pedúnculo acrescente después de la antesis. Sépalos con nervios comisurales notorios, verde oscuros. Cápsula coriácea, de igual largo que el cáliz, dehiscente por 10 dientes. Semillas reniformes, castaño-claras.

Escasa. Se distribuye en Chile de la I-II Región; también en Argentina, Bolivia y Perú. Exs.: Volcán Llullaillaco, 4640 m, enero 2004, V. Pardo 82 (CONC 158155).

\section{FABACEAE}

\section{Astragalus pusillus Vogel}

Planta cespitosa, subacaule, de 1,5-2,5 cm de alto. Hojas apretadas, seríceo-pilosas, pelos blancos algo hirsutos, lámina linear-lanceolada, de 1-2 cm de largo, folíolos pequeños, ovado-obovados, conduplicados. Flores dispuestas en espigas ovoideas den- 
sas. Corola púrpura. Legumbre ovado-oblonga a oblongo-elíptica, de 5-6 x $3 \mathrm{~mm}$, con pelos lanosos.

Escasa. Habita en la I-II Región; también en Argentina, Bolivia y Perú. Exs.: Volcán Llullaillaco, 4300 m, enero 2004, V. Pardo 104 (CONC 158156).

\section{MaLVACEAE}

8. Nototriche estipulata A.W.Hill ex B.L.Burtt Plantas cespitosa, tallo grueso. Hojas sin estípulas, pecíolos de ca. $1 \mathrm{~cm}$ de largo, lámina flabelada, de 2-3 x 4-6 mm, estrellado-pilosas, lóbulos 6-9, cortos, ápice café oscuro, carnoso. Cáliz estrellado-piloso. Corola estrellado-pilosa, pétalos obovados, de 3-4 x 1,5-2 mm. Carpelos rostrados, rostro y dorso seríceos.

Escasa. Endémica de la cordillera de la I-II Región. Exs.: Volcán Llullaillaco, 4500 m, marzo 2001, V. Pardo 30 (CONC 158153).

\section{Tarasa pediculata Krapov.}

Hierba anual, lámina trilobada o trífida, con pelos estrellados; estípulas filiformes. Inflorescencia en cincinos axilares, con 4-5 flores subsésiles, aglomeradas. Calículo de 2 bractéolas filiformes. Cáliz cubierto de pelos estrellados. Mericarpos de 8-10, dehiscentes, con 2 aristas de base ancha, con pelos largamente pediculados, de color negro, caras laterales lisas.

Escasa. Endémica de la II Región. Exs.: Quebrada Zorras, 4050 m, abril 2002, V. Pardo 59 (CONC 158154).

\section{ROSACEAE}

\section{Acaena magellanica (Lam.) Vahl}

"Amor seco, cadilla, trun".

Planta hasta $14 \mathrm{~cm}$ de alto. Hojas con 5-10 pares de folíolos, obovados, margen pinnatipartido, 47 pares de segmentos, glabros a pubescentes. Inflorescencia capituliforme, morada o amarilla. Cupela obcónica, tetrágona, con tricomas blandos, persistentes, blancos, con 4 espinas en el ápice.

Crece en los bordes de las vegas, escasa. Especie nativa distribuida a lo largo de todo el país. Crece también en Argentina. Exs.: Río Frío, 3820 $\mathrm{m}$, enero 2004, V. Pardo 106 (CONC 158162).

\section{SolanaceAe}

11. Jaborosa caulescens Gillies et Hook. var. bipinnatifida (Dunal) Reiche

Planta con tallos extendidos, con rizomas verticales u oblicuos. Hojas glabras, bipinnatisectas, con sus últimas divisiones dentado-mucronadas. Flores pequeñas. Cáliz con escasos tricomas glandulares y no glandulares. Corola con el tubo 2 veces más largo que el cáliz. Fruto violáceo, subgloboso, de 1-1,5 mm de diámetro.

Crece en los bordes de las vegas, escasa. Se distribuye desde la II-Región Metropolitana; también en Argentina. Exs.: Río Frío, 3900 m, diciembre 2001, V. Pardo 63 (CONC 158152).

\section{VERBENACEAE}

12. Glandularia origenes (Phil.) Schnack et Covas Planta robusta, glanduloso-híspida. Hojas dispuestas a la mitad del tallo, desnudos hacia el ápice. Hojas trapezoides, sésiles, trífidas, segmentos ovado-triangulares, agudos, enteros o tridentados, márgenes enroscados, cara inferior con nervios prominentes. Flores dispuestas en glomérulos multifloros. Cáliz prismático, con 5 dientes cortos. Tubo de la corola casi glabro, de color rosado pálido.

Crece en los bordes de las vegas, escasa. Habita entre la II-IV Región, también en la Argentina. Exs.: Quebrada Zorras, 3950 m, febrero 2003, V. Pardo 72 (CONC 158158).

\section{ANGIOSPERMAE: LILIOPSIDA POACEAE}

13. Calamagrostis chrysostachya (E. Desv.) Kuntze Plantas perennes, rizomatosas, de hasta $15 \mathrm{~cm}$. Vainas y láminas amarillentas, éstas de 2,5-10 cm, rígidas, curvadas, ápice punzante. Lígulas de 2,4-4,5 mm. Inflorescencias subespiciformes, de $1-5 \mathrm{~cm}$ de long. Espiguilla uniflora. Callo redondeado, pelos que no alcanzan el 1/3 inferior del antecio. Glumas agudas. Artejo de 0,3 mm . Lemmas de 4,4-5,5 mm, 5-nervia, escabrosas en la mitad superior, ápice hendido, arista dorsal retorcida, geniculada, escabrosa, de 6-6,5 mm. Abundancia media, crece en las vegas. Se distribuye desde la II, IV-RM y al oeste de Argentina (Mendoza y San Juan). Exs.: Río Frío, 3800 m, diciembre 2002, V. Pardo 66 (CONC 158151). 
14. Distichlis spicata (L.) Greene

"Grama salada, pasto salado".

Plantas dioicas, perennes, de 10-60 cm altura, con rizomas gruesos y ramificados, con escamas y punzantes. Lígulas breves, pestañosas. Láminas dísticas y rígidas, de 2,5-15 $\mathrm{cm}$, de ápices punzantes. Panículas espiciformes, espiguillas plurifloras, de 0,5$3 \mathrm{~cm}$. Panículas estaminadas de 8-9 cm, 6-30 espiguillas de 7-25 mm, 6-18-floras. Lemmas de 3,5$6 \mathrm{~mm}$. Anteras de 2-3 mm. Panículas pistiladas breves y densas, de $5-8 \mathrm{~cm}$, más de 35 espiguillas, de $10 \mathrm{~mm}$. Espiguillas 5-9-floras, fuertemente imbricadas. Lemmas coriáceas, de 3,5-6 mm, 7-11-nervias. Páleas carinas aladas y eroso-dentadas. Cariopsis de $2 \mathrm{~mm}$.

Abundante en los bordes de las vegas. En Chile habita entre las I-X Región, está además en Argentina, Bolivia y Perú. Exs.: Quebrada Zorras, 3560 m, febrero 2003, V. Pardo 73 (CONC 158159).

\section{Hordeum comosum J. Presl}

"Ratonera, cola de zorro, cebada patagónica". Plantas perennes, cespitosas, de 10-30 cm. Láminas planas a involutas, de hasta $10 \mathrm{~cm}$, pubescentes, aurículas. Lígula truncada, de 0,5 $\mathrm{mm}$. Espigas violáceas o verde amarillentas, semiincluidas de 4-9 $\mathrm{cm}$ long. Espiguillas unifloras, dispuestas en tríade, la central sésil, de 30-45 mm long. Glumas setiformes, de 25-40 mm, glabrescentes a escabrosas. Lemmas de 9-12 mm long., glabras. Páleas de 7,5-10 mm long., ápice apenas bidentado; raquilla de 2-6 mm. Anteras de $3 \mathrm{~mm}$ long. Espiguillas laterales pediceladas, de 1-2,5 mm long. Lemmas con aristas de 10-25 mm long. Glumas setáceas similares a las de la espiguilla central.

Crece en sitios áridos, en humedales y vegas de alta montaña. Habita desde la II-IX y XII Región, está presente en Argentina, Bolivia y Ecuador a lo largo de la Cordillera de los Andes. Exs.: Quebrada Zorras, 3450 m, febrero 2003, V. Pardo 71 (CONC 158157).

\section{Poa annua L.}

"Pasto de invierno, pastito de invierno".

Plantas anuales, cespitosas, de 5-30 cm. Láminas de hasta $15 \mathrm{~cm}$, tiernas, glabras. Lígula subaguda. Panojas piramidales, abiertas, laxas, de hasta $10 \mathrm{~cm}$. Espiguillas multifloras, de 3,5-8 mm, 3-8-floras. Glumas desiguales, la inferior aguda, 1-nervia, la superior obtusa, 3nervia, glabras, de anchos bordes membranáceos.
Lemmas de 2,8-4 mm, subagudas, 5-nervias, con anchos bordes membranáceos, pestañosas sobre los nervios en la mitad inferior. Páleas de 2-3 mm de quillas pestañosas. Anteras de 1-1,2 mm.

Adventicia con distribución cosmopolita, de origen europeo, frecuente en lugares removidos. Crece en todo Chile, Argentina, Bolivia y Perú. Exs.: Río Frío, 3750 m, enero 2004, V. Pardo 109 (CONC 158164).

\section{COMENTARIOS NOMENCLATURALES \\ RANUNCULACEAE}

Ranunculus exilis Phil.

Citada anteriormente como Ranunculus cymbalaria Pursh f. exilis (Phil.) Lourteig (Arroyo et al. 1998). Se trata de una especie muy distinta a $R$. cymbalaria, siendo, en general, plantas no mayores de 4,5 cm de alto, con las láminas de las hojas 3-5 lobadas a 3-5 partidas (Ruiz 2001).

\section{Poaceae}

Calamagrostis crispa (Rúgolo et Villav.) Govaerts

"Vizcachera, paja vizcachera, tembladera, sikuya". Citada anteriormente como Deyeuxia crispa Rúgolo et Villav. (Arroyo et al. 1998).

En pajonales áridos con suelos pedregosos, a alturas superiores a los $3800 \mathrm{~m}$ de altura. En Chile habita en la I y II Región. Se encuentra en Argentina, Bolivia y Perú.

Calamagrostis deserticola Phil. var. breviaristata (Rúgolo et. Villav.) Soreng

Citada anteriormente como Deyeuxia deserticola Rúgolo et Villav. (Arroyo et al. 1998).

En suelos semiáridos. En Chile se extiende en la I y II Región, está presente en el noroeste de Argentina y Bolivia.

Calamagrostis eminens J.Presl var. discreta (Rúgolo et Villav.) Soreng

Citada anteriormente como Deyeuxia eminens Rúgolo et Villav. (Arroyo et al. 1998).

En los bofedales (vegas) de las regiones altoandinas, entre los 3900-4500 $\mathrm{m}$ de altitud. En Chile se encuentra en la I y II Región. Crece en Argentina, Bolivia, Perú y Colombia. 
Calamagrostis eminens J.Presl var. fulva (Griseb.) Soreng

Citada anteriormente como Deyeuxia robusta Phil. (Arroyo et al. 1998).

Presente en Chile y Argentina (desde Jujuy hasta Mendoza). Vive en regiones andinas, entre los 3200$4300 \mathrm{~m}$ de altitud; se encuentra frecuentemente en sitios húmedos o vegas, próximos a las corrientes de agua.

Calamagrostis velutina (Nees et Meyen) Steud. Citada anteriormente como Deyeuxia velutina Nees et Meyen (Luebert \& Gajardo 1999).

En Chile habita en la II, III y IV Región; también en el oeste de Argentina.

Jarava frigida (Phil.) F. Rojas var. frigida "Paja amarilla"

Citada anteriormente como Stipa frigida Phil. (Arroyo et al. 1998).

Aparentemente es una especie común en el parque (Arroyo et al. 1998). En Chile se encuentra entre I-RM, VII Región. Crece además en la Puna de Perú y Bolivia entre los 3500-4300 m altura.

\section{DISCUSION}

Con las 16 nuevas adiciones aquí documentadas se tiene que la flora del parque comprende 126 especies, 74 géneros y 31 familias.

Arroyo et al. (1998) predijeron que la flora del parque podría incluir alrededor de las 110 especies, cifra que se alcanzaba con lo documentado por Luebert \& Gajardo (1999). Simonetti (1999) estimó, basado en la proporción de especies de Asteraceae presentes en el parque, que la flora total comprendería alrededor de 116 especies. Claramente con los nuevos hallazgos aquí reportados se ve que las predicciones subestimaron el tamaño de la flora del parque. Considerando además que el parque se ubica en una zona con altas fluctuaciones interanuales en las precipitaciones (Huston \& Hartley 2003), donde los ciclos pluviales asociados al fenómeno de El Niño cobran mucha relevancia en la emergencia de algunas especies, especialmente de las anuales (Latorre et al. 2003), no es de extrañar que se sigan reportando nuevas especies, incrementando la flora del Parque Nacional Llullaillaco.

Con los nuevos registros presentados se au- menta en 10 el registro de géneros respecto a lo documentado por Luebert \& Gajardo (1999). Los nuevos géneros son: Acaena, Azorella, Distichlis, Glandularia, Hordeum, Jaborosa, Poa, Polyachyrus, Silene y Tarasa. Entre las familias que aumentaron sus representantes están las Asteraceae con 3 especies, las Malvaceae con 2 especies y las Poaceae con 4 especies. Además se encontró un nuevo registro de familia para el parque, Rosaceae, con la especie Acaena magellanica, una de las especies que presentan mayor distribución en el género (Marticorena, en prensa).

Entre las especies nuevas reportadas se encuentran tres endémicas de Chile: Polyachyrus carduoides, Nototriche estipulata, Tarasa pediculata. Estas, sumadas a las 13 reportadas por Arroyo et al. (1998) y las cinco por Luebert \& Gajardo (1999), llevan el número de endémicas del parque a 21 , siendo un $16,6 \%$ de su flora. Este nivel de endemismo es mayor que el reportado por Arroyo et al. (1998) que indicaba un 14\%. Llama la atención que a pesar de la baja riqueza de especies, el nivel de endemismo en el PN Llullaillaco es mayor al encontrado en otras áreas silvestres protegidas localizadas en zonas de alta-montaña en Chile. A modo de comparación, señalamos que el porcentaje de especies endémicas en el Parque Nacional el Morado, ubicado en la cordillera de la Región Metropolitana, es de un 11\% (Teillier et al. 1994), mientras que la zona andina del Parque Nacional Torres del Paine no contiene especies endémicas de Chile, albergando sólo un 14,6\% de las especies nativas del extremo sur de Sudamérica (Arroyo et al. 1992).

A pesar de que el Parque Nacional Llullaillaco ha sido bastante explorado, este trabajo muestra que se siguen encontrando especies nuevas para el parque. Esto puede estar vinculado al hecho de la alta variabilidad interanual en el monto de las precipitaciones producto del fenómeno de El Niño. Futuras exploraciones debieran considerar este factor al momento de planificar los viajes y encontrar nuevos registros para la flora.

\section{AGRADECIMIENTOS}

Se agradece a Minera La Escondida por las facilidades otorgadas para la realización de los muestreos. Se agradece además el apoyo en terreno de Cristián Munizaga y Oscar Zepeda. Este manuscrito se es- 
cribió en el marco del Proyecto Vicuña, desarrollado por la gerencia de Medio Ambiente de Minera Escondida Ltda.

\section{BIBLIOGRAFIA}

Arroyo, M.T.K., Von Bohlen, C., Cavieres, L. \& Marticorena, C. 1992. Survey of the alpine flora of Torres del Paine National Park, Chile. Gayana Botanica 49: 47-70.

Arroyo, M.T.K., Castor, C., Marticorena, C., Muñoz, M., Cavieres, L., Matthei, O., Squeo, F., Grosjean, M. \& Rodríguez, R. 1998. The flora of Llullaillaco National Park located in the transitional winter-summer rainfall area of the northern Chilean Andes. Gayana Botanica 55: 93 110.

Huston, J. \& Hartley, A.J. 2003. The central Andean west-slope rainshadow effect and its potential contribution to the origin of hyper-aridity in the
Atacama desert. International Journal of Climatology 23: 1453-1464.

Latorre, C., Betancourt, J.L., Rylander, K.A., Quade, J. \& MAtтehi, O. 2003. A vegetation history from the arid prepuna of northern Chile $\left(22-23^{\circ} \mathrm{S}\right)$ over the last 13500 years. Palaeography, Palaeoclimatology, Palaeoecology 194: 223-246.

LUEBERT, F. \& GAJARDO, R. 1999. Adiciones y notas a la flora del Parque Nacional Llullaillaco (Región de Antofagasta, Chile). Noticiero Mensual del Museo Nacional de Historia Natural 339: 3-6.

Marticorena, A. (en prensa). Revisión del género Acaena (Rosaceae) en Chile. Annals of the Missouri Botanical Garden.

Ruiz, E. 2001. Ranunculaceae. En C.Marticorena y R.Rodríguez, Flora de Chile Vol.2, Fasc. 1. 40-84.

Simonetti, J.A. 1999. On the size of the Chilean Flora (a speculation). Journal of Mediterranean Ecology 1: 129-132.

Teillier, S., Hoffmann, A., Saavedra, F. \& Pauchard, I. 1994. Flora del Parque Nacional El Morado, Región Metropolitana, Chile. Gayana Botanica 51: $13-47$.

Fecha de recepción: 13.07.04

Fecha de aceptación: 11.08.04 